\title{
LAMINATED WORKSHEETS AS A LEARNING RESOURCE FOR STUDENTS IN THEATRE
}

Rollin M, Vithlani R, Brewer CF, Pieper N. Imperial College London and Imperial College Healthcare, London UK

Medical students must gain surgical experience

This impacts upon their career decisions $\mathbf{s}^{1,2}$

Perceived value of theatre experience can be low ${ }^{3}$

Negative factors 4,5 include:

inter-team variability

poor visibility

unclear learning objectives

approachability of staff

\section{Increased interaction leads to greater reported quality of teaching and satisfaction'.}

Student inactivity in theatre is a lost learning opportunity. Literature regarding improvement of student experience in the operating theatre is sparse.

\section{METHODS}

Laminated worksheets introduced \& evaluated for tonsillectomy, adenoidectomy, grommets, myringoplasty, septoplasty, FESS, ML, Paeds MLB.

Worksheet sections:

Anatomy

Physiology

Pathology

Surgical steps

Key moments

Instruments

Potential complications

Considerations for recovery

Three questions for students to discuss
Student evaluation of worksheets using Likert scales and free text responses. 24 responses received.

RESULTS

87\% agreed the worksheets facilitated learning, 3 neutral opinions

$79 \%$ agreed the content of the sheets was useful, 4 neutral opinions, 1 strongly disagree

$83 \%$ agreed the sheets enabled them to meet learning objectives, 4 neutral opinions

\section{Free-text response themes:}

Facilitated learning

Opportunity to read about other procedures

Font size too small

Useful to read before a case

Useful to read before the list
DISCUSSION

Worksheets are useful, and make use of student down-time

Students read about other procedures that they couldn't see that day

We would prefer first-hand experience, but secondhand experience is better than nothing

Worksheets are designed for two sides of A4

Advantages of worksheets: Simple Easy to produce Easy to distribute Wipe-clean when laminated

Electronic worksheets would be simple, but easy availability might lead to students not attending theatre, erroneously considering the worksheets an acceptable substitute.

\section{Student inactivity in theatre is a lost learning opportunity}

This approach could be adapted for other surgical specialties and disciplines, if felt to be useful.

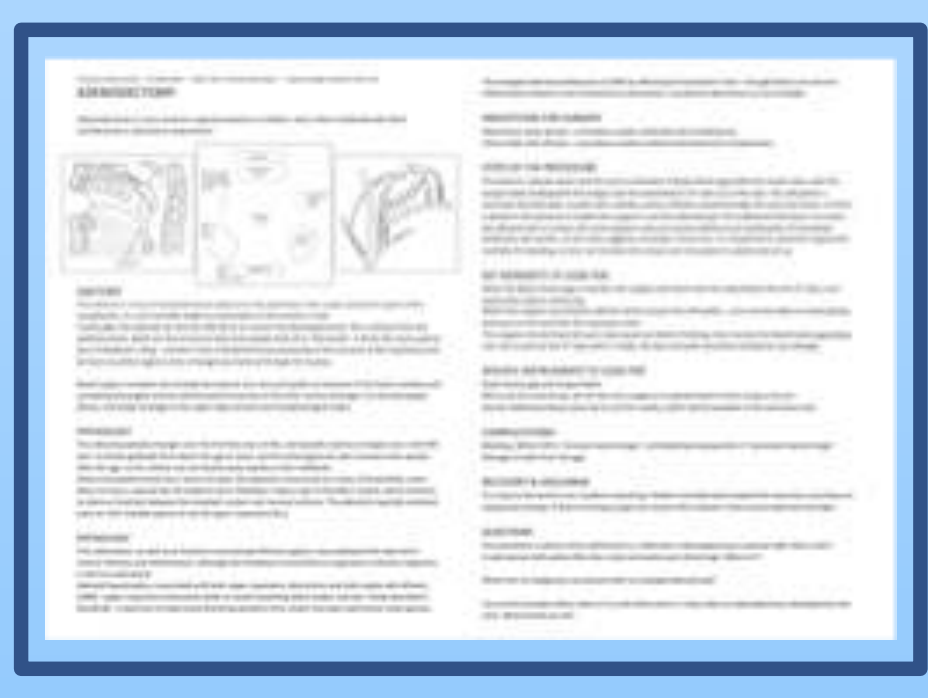

LIMITATIONS

Small sample size - we are still collecting data

Unable to access examination results to examine for potential correlation

\section{The worksheets facilitate learning}

\section{The content of the worksheets is useful}

The worksheets enabled me to meet learning objectives

"Read the sheets beforehand - good for planning what to look out for and learning objectives"

"Anatomy and physiology were explained well"

"Theatre sheets were very useful for pre-reading and during surgery"

"Operating theatre sheets are a useful teaching tool"

"The best part was the teaching which was tailored to the sheets. The booklet was very informative" 